\title{
A New Approach for Applying Support Vector Machines in Multiclass Problems Using Class Groupings and Truth Tables
}

\author{
Mauricio Kugler, Hiroshi Matsuo and Akira Iwata \\ mauricio@kugler.com,matsuo@nitech.ac.jp, iwata@nitech.ac.jp \\ Department of Computer Science and Engineering \\ Nagoya Institute of Technology \\ Gokiso-cho, Showa-ku, 466-8555, Nagoya, Japan
}

The Support Vector Machines (SVMs) had been showing a high capability of complex hyperplane representation and great generalization power. These characteristics lead to the development of more compact and less computational complex methods than the One-versus-Rest $(\mathrm{OvR})$ and One-versus-One $(\mathrm{OvO})$ [1] classical methods in the application of SVMs in multiclass problems. This paper proposes a new method for this task, named Truth Table Fitting Multiclass $S V M$ (TTF-MCSVM), in which less SVMs are used than other classical methods. The main objective of this research is the development of a new method to be applied in problems with very large number of classes, like in the recognition of East Asian languages characters (e.g. Japanese and Chinese kanji).

The TTF-MCSVM is based on the combination of many simple binary SVMs, like the OvR and OvO. The $N$ classes are divided in $M$ combinations of two groups, where $M$ is:

$$
M=\left\lceil\log _{2} N\right\rceil
$$

Each of the two groups in each combination will correspond to a new pseudoclass and each pair of pseudo-classes forms a grouping. As the $M$ groupings are binary, they can be applied to $M$ simple and independent binary SVM. This approach can be also represented by the encoding scheme described in [2]. However, the principal point in this work is to analyze the use of encodings with a fixed length $M$, which is the minimal encoding size for a problem with $N$ classes. Considering the classes $C_{k}(1<k<N)$, if the groupings are consistent (i.e. each $C_{k}$ class has a different associated pseudo-class combination), the $M$ groupings can be arranged in a Boolean truth table, where the inputs are the results of all SVMs and the output is the winner class, which is chosen by the overlap of the decision hyperplanes of the SVMs. During the training phase, each SVM is trained independently, and the margin is maximized to divide the two pseudo-classes of that SVM. On the test phase, the results of the $M$ SVMs are applied on the truth table and, if the groupings are correct, it will be possible to choose the winner class.

A very basic example is shown in Fig. 1. The same 9 class problem was solved using the TTF-MCSVM (Fig. 1(a), with the groupings drawn in the figure), OvR (Fig. 1(b)) and OvO (Fig. 1(c)) methods. All of them used gaussian kernel with $\sigma=1.5$ and $C=100$. The problem could be solved by the TTF-MCSVM 


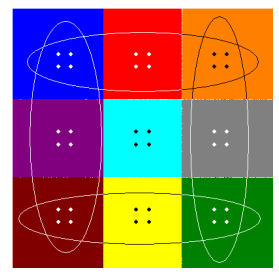

(a)

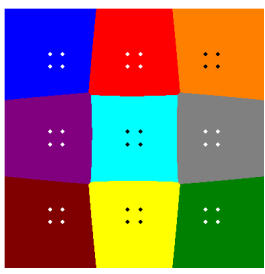

(b)

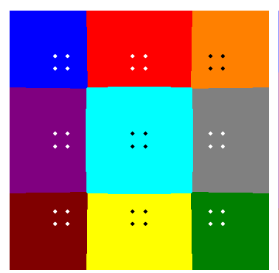

(c)

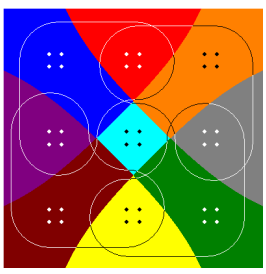

(d)

Fig. 1. Nine classes problem: (a) TTF-MCSVM (3 SVMs, 16 SV, Grouping Set A), (b) OvR (8 SVMs, 47 SV), (c) OvO (28 SVMs, 108 SV), (d) TTF-MCSVM (3 SVMs, $20 \mathrm{SV}$, Grouping Set B)

with less classifiers, less found support vectors (SV) and presenting a better generalization than the other methods. However, as can be seen in (Fig. 1(d)), a different grouping set gives a visible smaller margin than Fig. 1(a).

Table 1 shows the comparison of three UCI benchmark problems, iris, wine and glass, solved by the TTF-MCSVM (with random groupings), OvR and OvO methods. The results shows the smaller number of support vectors for the best performance parameters combinations, calculated over the average of a 10-fold cross validation ( $p$ means $d$ for polynomial kernel and $\sigma$ for gaussian kernel).

Table 1. UCI benchmark experiments comparison

\begin{tabular}{|c|c|c|c|c|c|c|c|}
\hline \multirow{2}{*}{$\begin{array}{l}\text { Problem } \\
(N, \text { samples })\end{array}$} & \multicolumn{3}{|c|}{ TTF-MCSVM } & \multicolumn{2}{|l|}{ OvR } & \multicolumn{2}{|l|}{$\mathrm{OvO}$} \\
\hline & $M \mathrm{CR} \%$ & $\# \mathrm{SV}$ & $p, C$ & $M \mathrm{CR} \% \# \mathrm{SV}$ & $p, C$ & $M \mathrm{CR} \% \# \mathrm{SV}$ & $p, C$ \\
\hline \multicolumn{8}{|c|}{ Polynomial Kernel } \\
\hline iris $(3,150)$ & 299.33 & 31 & $2,1.0$ & $\begin{array}{|lll|}3 & 99.33 & 52 \\
\end{array}$ & $2,0.9$ & 394.67 & $2,0.03$ \\
\hline wine $(3,178)$ & 298.82 & 50 & $1,0.2$ & $\begin{array}{lll}3 & 98.82 & 106\end{array}$ & $2,0.07$ & 398.82 & $1,0.2$ \\
\hline glass $(6,214)$ & 366.19 & 304 & $2,0.2$ & $\begin{array}{lll}6 & 69.52 & 844\end{array}$ & $3,0.004$ & $1570.95 \quad 502$ & $2,0.02$ \\
\hline \multicolumn{8}{|c|}{ Gaussian Kernel } \\
\hline iris $(3,150)$ & 298.67 & 19 & 4,80 & $\begin{array}{|lll|}3 & 98.67 & 43\end{array}$ & 3,60 & 396.00 & 1,1 \\
\hline wine $(3,178)$ & 298.82 & 49 & 20,100 & 399.41 & 10,9 & 399.41 & 10,3 \\
\hline glass $(6,214)$ & 375.71 & 363 & $0.9,4$ & $\begin{array}{lll}6 & 75.71 & 603\end{array}$ & 1,6 & $1576.19 \quad 560$ & 1,4 \\
\hline
\end{tabular}

The TTF-MCSVM performance had shown no statistically significant difference from the OvR and $\mathrm{OvO}$ methods, using a smaller number of classifiers and, in almost all cases, considerably less SV. In the case of the glass problem, optimized groupings sets could increase the performance. The small number of classifiers, specially when the kernel matrix is calculated in advance, leads to smaller training time. Future works include the study about the groupings optimization and experiments with more complex real world problems.

\section{References}

1. Jason Weston and Chris Watkins. Support vector machines for multi-class pattern recognition. In Proceedings of the 7th European Symposium On Artificial Neural Networks, pages 219-224, Bruges, 41999.

2. Thomas G. Dietterich and Ghulum Bakiri. Solving multiclass learning problems via error-correcting output codes. Journal of Artificial Intelligence Research, 2:263-286, 1995. 